[11] L. Gillman and M. Jerison, Rings of continuous funotions, New York 1960.

[12] P. R. Halmos, Injective and projective Boolean Algebras, in Proc. Symp. Pure Math. II, Lattice theory, Providence 1961, pp. 114-122.

[13] - Lectures on Boolean Algebras, New York 1963.

[14] E. Hewitt, A remark on density characters, Bull. Amer. Math. Soc. 52 (1946), pp. 641-643.

[15] C. Kuratowski, Topologie I, Warszawa 1958

[16] E. Marczewski (E. Szpilrajn), Remarque sur les produits cartésiens d'espaces topologiques, Dokl. Akad. Nauk SSSR, 31 (1941) pp. 525-528.

[17] - Séparabilité et multiplication cartésienne des espaces topologiques, Fund. Math. 34 (1947), pp. 127-143.

[18] E. S. Pondiczery, Power problems in abstraet spaces, Duke Math. Journ. 11 (1944), pp. 835-837.

[19] K. A. Ross and A. H. Stone, Products of separable spaces, Amer. Math. Monthly 71 (1964), pp. 398-403.

[20] N. Sanin, On products of topological spaces, Trudy Mat. Inst. Steklova 24 (1948) (Russian).

[21] N. Vedenissoff, Remarques sur la dimension des espaces topologiques, Uě. Zapiski Mosk. Gos. Univ. 30 (1939), 131-140 (Russian; French summary).

INSTYTUT MATEMATYCZNY POLSKIEJ AKADEMII NAUK

INSTITUTE OF MATHEMATICS OF THE POLISH ACADEMY OF SCIENCES

Reģu par la Rédaction le 22. 12. 1964

\section{Ordnungsfähigkeit zusammenhängender Räume}

\author{
von \\ H. Herrlich (Berlin)
}

Ziel der Arbeit ist eine topologische Kennzeichnung der zusammenhängenden (Satz 1) und der lokal-zusammenhängenden (Satz 2, 2a) ordnungsfähigen Räume.

DEFTNITIONEN.

1) Ein topologischer Raum $T$ heißt ordnungsfähig, wenn er einem geordneten Raum homöomorph ist, d.h. wenn es eine lineare Ordnung $R$ auf $T$ so gibt, daß die offenen Intervalle eine Basis der Topologie von $T$ bilden.

2) Ein Punkt $x$ einer zusammenhängenden Menge $M$ heißt Randpunkt von $M$, wenn $M-\{x\}$ zusammenhängend ist, sonst Schnittpunkt von $M$.

3) Ein topologischer Raum heißt randendlich, wenn jede seiner zusammenhängenden Teilmengen höchstens zwei Randpunkte enthält.

HILFSSÄTZE.

1) Sind $x, y$ zwei verschiedene Elemente des zusammenhängenden, lokal-zusammenhängenden $T_{1}$-Raumes $T$, so gibt es eine Komponente $K$ von $T^{\prime}=T-\{x, y\}$, die $x$ und $y$ als Häufungspunlte besitzt. $K_{1}=K \cup\{x, y\}$ ist als Unterraum lokal-zusammenhängend.

Beweis: a) $\mathfrak{M}$ sei die Menge aller Komponenten von $T^{\prime}$. Jedes $K$ aus $\mathfrak{M}$ ist offen-abgeschlossen in $T^{\prime}$. Gäbe es ein $K$ in $\mathfrak{M}$, das weder $x$ noch $y$ als Häufungspunkt besäße, so wäre dieses $K$ offen-abgeschlossen in $T$, im Widerspruch zum Zusammenhang von $T$. Jedes $K$ aus $\mathfrak{M}$ besitzt also $x$ oder $y$ als Häufungspunkt. Ist $U$ eine zu $y$ disjunkte, zusammenhängende Umgebung von $x$ und enthält ein $K$ aus $\mathfrak{M}$ Elemente von $U$, so ist $x$ Häufungspunkt von $K$; denn sonst wäre $K \frown U$ offen-abgeschlossen in $U$. Also umfaßt $X=\bigcup\left\{K \mid K \in \mathfrak{M}, x \in K^{-}\right\} \cup\{x\}$ die Menge $U$, ist somit Umgebung von $x$, also offen in $T$. Analog ist $Y=\bigcup\{K \mid K \in \mathfrak{M}$, $y \in K\} \cup\{y\}$ offen in $T$. Hätte keine Komponente $x$ und $y$ als Häufungspunkte, so wären $X, Y$ disjunkt, im Widerspruch zum Zusammenhang von $T$. 
b) $K$ ist offen in $T$, also lokal-zusammenhängend. Ist $Z$ eine zu $y$ disjunkte, offene, zusammenhängende Umgebung von $x$, so ist $Z \cap K_{1}$ zusammenhängend; denn wäre letzteres nicht der Fall, so gäbe es eine Komponente $L$ von $Z \cap K_{1}$, die $x$ nicht enthielte, also in $Z$ offen-abgeschlossen wäre, im Widerspruch zum Zusammenhang von $Z$. Somit besitzt auch $x$ eine Basis aus zusammenhängenden Umgebungen in $K_{1}$. Ebenso $y$. $K_{1}$ ist lokalzusammenhängend.

2) Ist $x$ Schnittpunkt des zusammenhängenden, lokal-zusammenhängenden, randendlichen $T_{1}$-Raumes $T$, so besteht $T-\{x\}$ aus genau zwei Komponenten.

Bew eis. Wären $K_{1}, K_{2}, K_{3}$ verschiedene Komponenten von $T-\{x\}$, $x_{i}$ Elemente von $K_{i}$ für $i=1,2,3$, so gäbe es nach H. S. 1 Komponenten $M_{i}$ von $T-\left\{x, x_{i}\right\}$, mit $\left\{x, x_{i}\right\} \subset M_{i}^{-}$. Also wären $x_{1}, x_{2}, x_{3}$ Randpunkte der zusammenhängenden Menge $\bigcup_{i=1}^{8} M_{i} \cup\left\{x, x_{1}, x_{2}, x_{3}\right\}$, im Widerspruch zur Randendlichkeit von $T$.

3) Besitzt der zusammenhängende, lokal-zusammenhängende, randendliche $T_{1}$-Raum $T$ die Randpunkte $a, b$ und ist $x$ Schnittpunkt von $T$, so besteht $T-\{x\}$ aus genau zwei Komponenten, von denen eine $a$, die andere $b$ enthält.

Beweis. $T^{\prime}=T-\{a\}$ ist ein zusammenhängender, lokal-zusammenhängender, randendlicher $T_{1}$-Raum, $T^{\prime}-\{x\}$ besteht also nach H. S. 2 aus genau zwei Komponenten $L_{1}, L_{2}$ mit $a \in L_{1}^{-}$. Folglich sind $K_{1}=L_{1} \cup\{a\}$, $K_{2}=L_{2}$ zusammenhängend, also die Komponenten von $T-\{x\}$. Da $L_{1}$ zusammenhängend ist, muß a Randpunkt von $K_{1}$ sein. Wäre $b$ ebenfalls in $K_{1}$ enthalten, so könnte man analog zeigen, daß auch $b$ Randpunkt von $K_{1}$ wäre. Dann wären $a, b, x$ Randpunkte von $K_{1} \cup\{x\}$, im Widerspruch zur Randendlichkeit von $T$. Also ist $b$ Element von $K_{2}$.

4) Besitzt der zusammenhängende $T_{1}$-Raum $T$ genau zwei Randpunkte $a, b$ und besteht $T-\{x\}$ für jeden Schnittpuntet $x$ aus zwei separierten Mengen $A(x), B(x)$ mit $a \in A(x), b \in B(x)$, so gibt es eine lineare Ordnung $R$ auf $T$ derart, daß die Ordnungstopologie gröber ist als die gegebene Topologie von $T$.

Beweis. Man setze $A(a)=B(b)=\emptyset, A(b)=B(a)=T$. Dann ist $R=\{(x ; y) \mid x \in A(y)\}$ eine lineare Ordnung auf $T$; denn:

1a) Ist $x$ Element von $A(y)$, so ist $B(y) \cup\{y\}$ zusammenhängend in $T-\{x\}$, also in $B(x)$ enthalten.

1b) Ist $x$ Element von $B(y)$, so ist $A(y) \cup\{y\}$ zusammenhängend in $T-\{x\}$, also in $A(x)$ enthalten.

2) Es gelt $x R y, y R z$. Dann folgt aus 1a) $B(y) \cup\{y\} \subset B(x), B(z) \cup\{z\}$ $C B(y)$, also $z \in B(x)$, hieraus mit Hilfe von 1b) $x \in A(z)$, also $x R z$. $R$ ist transitiv.
3) Sei $x \neq y, x \notin A(y)$. Dann gilt $x \in B(y)$, also nach 1 b) $y \in A(x)$. Es gilt wenigstens eine der drei Relationen $x R y, y R x, x=y$.

4) Es gilt höchstens eine dieser drei Relationen, weil $x R x$ für kein $x$ gilt.

Also is $R$ eine lineare Ordnung auf $T$. Die Nengen $A(x), B(x)$ bilden eine Subbasis der zugehörigen Ordnungstopologie. Da sie sämtlich in $T$ offen sind, ist die Ordnungstopologie gröber als die Topologie von $T$.

5) $(T, \mathfrak{T})$ sei ein topologischer Raum und $R$ eine lineare Ordnung auf $T$, so daß die Ordnungstopologie $\mathfrak{I}_{R}$ gröber als die Topologie $\mathfrak{I}$ ist.

a) Ist $(T, \mathfrak{I})$ kompakt, so gilt $\mathfrak{T}=\mathfrak{T}_{R}$.

b) Ist $(T, \mathfrak{I})$ zusammenhängend und lokal-zusammenhängend, so gilt $\mathfrak{I}=\mathfrak{I}_{R}$.

Beweis. a) Die Identität auf $T$ ist eine bijektive, stetige Abbildung des kompakten Raumes $(T, \mathfrak{T})$ auf den $T_{2}$-Raum $\left(T, \mathfrak{I}_{R}\right)$ und somit ein Homöomorphismus.

b) Die Menge $\mathfrak{B}$ aller bezüglich $\mathfrak{I}$ zusammenhängenden, offenen Mengen bildet eine Basis von $\mathfrak{I}$. Jedes $B$ aus $\mathfrak{B}$ ist notwendig ein Intervall bezüglich $R$. Wäre ein derartiges $B$ bezüglich $\mathfrak{I}_{R}$ nicht offen, so gäbe es ohne Beschränkung der Allgemeinheit ein $x$ in $B$ derart, daß die Menge $M(x)=\{y \mid x R y\}$ nicht leer und zu $B$ disjunkt, also bezüglich $\mathfrak{I}$ offenabgeschlossen wäre, im Widerspruch zum Zusammenhang von $(T, \mathfrak{T})$. Also ist jedes $B$ aus $\mathfrak{B}$ offen bezüglich $\mathfrak{I}_{R}$ und somit $\mathfrak{I} \subset \mathfrak{I}_{R}$, also $\mathfrak{I}=\mathfrak{T}_{R}$

6) (Eindeutigkeit der Ordnung): Ist der zusammenhängende Raum $T$ durch $R$ und durch $S$ zulässig geordnet, so gilt $R=S$ oder $R=S^{-1}$.

Beweis. Für $A \subset T$ sei $R / A$ bzw. $S / A$ die Einschränkung von $R$ bzw. $S$ auf $A$. Die Menge $\mathfrak{M}=\{A|A \subset T, R / A=S| A\}$ wird durch Inklusion induktiv geordnet, besitzt also maximale Elemente. Sind alle maximalen Elemente von $\mathfrak{M}$ höchstens einpunktig, so gilt $R=S^{-1}$. Andernfalls gibt es ein maximales $A$ in $\mathfrak{M}$, welches wenigstens zwei Punkte enthält. Gäbe es ein $x$ in $T-A$, so gäbe es ohne Beschränkung der Allgemeinheit ein $y$ in $A$ mit $x R y, y S x$. Sei $z$ ein Element von $A-\{y\}$.

1. Fall: $y R z$. Dann liegen $x$ und $z$ bezüglich $R$ in verschiedenen, bezüglich $S$ in derselben Komponente von $T-\{y\}$. Widerspruch.

2. Fall: $z R y$. Dann liegen $x$ und $z$ bezüglich $R$ in derselben, bezüglich $S$ in verschiedenen Komponenten von $T-\{y\}$. Widerspruch.

Folglich gilt $A=T$, also $R=S$.

7) Sind $A, B$ zwei durch $R$ beziehungsweise $S$ zulässig geordnete, zusammenhängende Teilräume des randendlichen Raumes $T$ und besitzen $A$ und $B$ bezüglich $R$ beziehungsweise $S$ denselben Anfangspunkt $a$ und denselben Endpunkt $b$, so gilt $A=B$ und $R=S$. 
Beweis. Wäre $x$ Element von $A-B$, so wären $a, b, x$ Randpunkte von $A \cup B$, im Widerspruch zur Randendlichkeit von $T$. Also $A \subset B$. Analog $B C A$, somit $A=B$. Nach H. S. 6 muß dann auch $R=S$ gelten.

8) Sind $A$ und $B$ zwei zusammenhängende, durch $R$ beziehungsweise $S$ zulässig geordnete Teilräume des randendlichen Raumes $T$ und ist $i \mathrm{hr}$ Durchschnitt $D$ nicht leer, so ist auch ihre Vereinigung $V$ ordnungsfähig.

Beweis. Sind $x$ und $y$ Elemente von $D$, so gehören nach H. S. 7 auch die von $x, y$ aufgespannten Teilintervalle von $A$ beziehungsweise $B \mathrm{zu} D$. Also ist $D$ ein Intervall bezüglich $A$ und bezüglich $B$, somit zusammenhängend und nach H. S. 6 kann man ohne Beschränkung der Allgemeinheit.annehmen, daß die Einschränkungen von $R$ auf $D$ und von $S$ auf $D$ identisch sind.

Also:

$$
\begin{array}{cccc}
A=A_{1} \cup D \cup A_{2} & \text { mit } & A_{1} \times D \subset R, & D \times A_{2} \subset R . \\
B=B_{1} \cup D \cup B_{2} & \text { mit } & B_{1} \times D \subset S, & D \times B_{2} \subset S .
\end{array}
$$

Sind $a_{1}, a_{2}, b_{1}, b_{2}$ Elemente von $A_{1}, A_{2}, B_{1}, B_{2}$, so sind sie Randpunkte von $\left[a_{1}, a_{2}\right]_{R} \cup\left[b_{1}, b_{2}\right]_{s}$. Wegen der Randendlichkeit von $T$ müssen also zwei der vier Mengen $A_{1}, A_{2}, B_{1}, B_{2}$ leer sein. Enthält $D$ nur einen Punkt, so folgt hieraus sofort die Ordnungsfähigkeit von $A \cup B$. Enthält $D$ mehr. als einen Punkt, so schließt man zunächst wie oben, daß eine der Mengen $A_{1}, B_{1}$ und eine der Mengen $A_{2}, B_{2}$ leer sein muß, woraus ebenfalls die Ordnungsfähigkeit von $A \cup B$ folgt.

SATZ 1. Ein zusammenhängender Raum ist genau dann ordnungsfähig, wenn er ein lokal-zusammenhängender, randendlicher $T_{1}$-Raum ist.

Beweis. a) Die Bedingungen sind offenbar notwendig.

b) $T$ erfülle die Bedingungen. Die Menge $\mathfrak{M}$ aller zusammenhängenden, ordnungsfähigen Teilräume von $T$ wird durch Inklusion teilweise geordnet. Ist $I$ eine wohlgeordnete Indexmenge mit dem Anfangselement 1 und $f$ eine monoton steigende Abbildung von $I$ in $\mathfrak{M}$, so ist $F=\bigcup\{f(i) \mid i \in I\}$ wieder zusammenhängend. Sei $f(1)$ ohne Beschränkung der Allgemeinheit mehr als einpunktig, $R_{1}$ eine zulässige Ordnung von $f(1)$. Dann gibt es wegen H. S. 6 zu jedem $i$ aus $I$ genau eine zulässige Ordnung $R_{\mathfrak{k}}$ von $f(i)$, deren Einschränkung auf $f(1)$ gleich $R_{1}$ ist. Wegen der Randendlichkeit von $T$ ist $R=\bigcup\left\{R_{i} \mid \dot{i} \in I\right\}$ eine zulässige Ordnung von $\boldsymbol{F} . \boldsymbol{F}$ ist somit Element von $\mathfrak{M}, \mathfrak{M}$ also induktiv geordnet und besitzt folglich ein maximales Element $M$. Wäre $M$ von $T$ verschieden, so könnte man ein $x$ in $M$ und ein $y$ in $T-M$ und nach H. S. 1 eine Komponente $K$ von $T-\{a, y\}$ so wählen, daß $x, y$ Häufungspunkte von $K$ wären. Nach H. S. $1,2,3,4,5 \mathrm{~b}$ wäre $L=K \cup\{x, y\}$ ordnungsfähig, somit nach H.S. 8 auch $L \cup M$, im Widerspruch zur Maximaleigeñschaft von $M$. Also $M=T$. $T$ ist ordnungsfähig.
SATz 1a (Kowalsky). Ein zusammenhängender Raum $T$ ist genau dann ordnungsfähig, wenn er ein lokal-zusammenhängender $T_{\mathrm{I}}$-Raum ist und wenn es unter je drei echten, zusammenhängenden Teilmengen von $T$ stets zwei gibt, die $T$ nicht überdecken.

Beweis. a) Die Bedingungen sind offenbar notwendig.

b) Sind die Bedingungen erfüllt, so ist $T$. randendlich; denn wären $x_{1}, x_{2}, x_{3}$ verschiedene Randpunkte einer zusammenhängenden Menge $Z$ von $T$ und wäre für $i=1,2,3 K_{i}$ diejenige Komponente von $T-\left\{x_{i}\right\}$, die $Z-\left\{x_{i}\right\}$ umfaßt, so wären die $K_{i}$ offen in $T$, die $K_{i} \cup\left\{x_{i}\right\}$ abgeschlossen in $T$, also für $i \neq k \quad K_{i} \cup K_{k}=K_{i} \cup\left\{x_{i}\right\} \cup K_{k} \cup\left\{x_{k}\right\}$ offen-abgeschlossen in $T$ und somit gleich $T$, im Widerspruch zu den Voraussetzungen. $T$ ist randendlich, also nach Satz 1 ordnungsfähig.

Bemerkung. Eine andersartige Kennzeichnung der zusammenhängenden, ordnungsfähigen Räume stammt von Eilenberg.

BeIspIELe. 1) Der durch $T=\left\{(x ; y\} \mid x>0, y=\sin \left(x^{-1}\right)\right\} \cup\{(0 ; 0)\}$ bestimmte Unterraum des 2-dimensionalen Euklidischen Raumes $R^{2}$ ist ein randendlicher, zusammenhängender metrischer Raum, der nicht lokal-zusammenhängend also nicht ordnungsfähig ist.

2) $\mathfrak{I}_{0}$ sei die natiürliche Topologie des 3-dimensionalen Euklidischen Raumes $R^{3} . T$ sei die Menge aller Punkte $(x ; y ; z)$ des $R^{3}$ mit $z \geqslant 0$. Bezeichnet man für reelle $x, y$ mit $g(x, y)$ die Menge $\{(x ; y ; z) \mid z>0\}$, so bildet $\mathfrak{S}=\{g(x, y) \mid x \in R, y \in R\} \cup\left\{B \cap T \mid B \in \mathfrak{T}_{0}\right\}$ eine Subbasis einer Topologie $\mathfrak{I}$ auf $T$. Der so konstruierte Raum $(T, \mathfrak{I})$ hat folgende Eigenschaften:

1. $(T, \mathfrak{I})$ ist metrisierbar. Bezeichnet man mit $\varrho$ die Euklidische Metrik des $R^{3}$, so wird z.B. durch

$\bar{\varrho}((x ; y ; z),(\bar{x} ; \bar{y} ; \bar{z}))=\left\{\begin{array}{l}\varrho((x ; y ; z),(\bar{x} ; \bar{y} ; \bar{z}))+|z-\bar{z}|, \text { wenn }(x ; y)=(\bar{x} ; \bar{y}), \\ \varrho((x ; y ; z),(\bar{x} ; \bar{y} ; \bar{z}))+|z+\bar{z}|, \text { wenn }(x ; y) \neq(\bar{x} ; \bar{y})\end{array}\right.$ eine mit $\mathfrak{I}$ verträgliche Metrik auf $T$ definiert.

2. $(T, \mathfrak{T})$ is zusammenhängend und lokal-zusammenhängend.

3. Für jedes $x$ aus $T$ zerfällt $T-\{x\}$ in genau zwei Komponenten.

4. $(T, \mathfrak{I})$ ist nicht randendlich, also nicht ordnungsfähig. Durch dieses Beispiel wird eine bei Bourbaki (S. 195, Ex. 15b) aufgestellte Behauptung widerlegt.

Die topologische Summe ordnungsfähiger Räume ist offenbar unterordnungsähig, d.h. einem Unterraum eines ordnungsfähigen Raumes homöomorph. Jeder lokal-zusammenhängende Raum ist topologische Summe zusammenhängender Räume. Also gilt:

SATZ 2. Ein lokal-zusammenhängender Raum ist genau dann unterordnungstähig, wenn er ein randendlicher $T_{1}$-Raum ist. 
Andererseits ist ein derartiger Raum im allgemeinen nicht ordnungsfähig, wie der durch $T=(0,1) \cup\{2\}$ erzeugte Unterraum des 1-dimensionalen Euklidischen Raumes $R^{1}$ zeigt.

HWFSSATZ 9. $I_{0}, I_{1}, I_{2}$ seien drei parweise disjunkte Indexmengen und jedem $i$ aus $I=I_{0} \cup I_{1} \cup I_{2}$ sei ein topologischer Raum $T_{i}$ und eine zulässige Ordnung $R_{i}$ auf $T_{i}$ so zugeordnet, daß jedes $T_{i}$ bezüglich $R_{i}$ für $i \in I_{0}$ weder einen Anfangs- noch einen Endpunkt, für $i \in I_{1}$ entweder einen Anfangs- oder einen Endpunkt, für $i \in I_{2}$ einen Anfangs- und einen Endpuntet besitzt. Ist eine der vier folgenden Bedingungen erfïllt, so ist die topologische Summe $T$ der $T_{i}, i \in I$ ordnungsfähig:
(a) $I_{0}=\varnothing$,
(b) $I_{2}=\emptyset$,
(c) $I_{1} \neq \varnothing$
(d) $\left|I_{2}\right| \geqslant \mathrm{s}_{0}$.

Beweis. Man nenne eine lineare Ordnung $R$ auf einer Menge $M$ diskeret, wenn $M$ bezüglich der durch $R$ induzierten Topologie ein diskreter Raum ist. Dann gibt es zu jeder Menge $M$ diskrete Ordnungen (Ist z.B. $|M|=\kappa_{a}$ und $\mu=\left(\omega_{0}^{-1}+\omega_{0}\right)$ der Ordnungstyp der ganzen Zahlen, so kann man eine Ordnung auf $M$ z.B. so wählen, daß der zugehörige Ordnungstyp gleich einem der folgenden Ordnungstypen ist: $\mu \cdot \omega_{a}, \omega_{0}+\mu \cdot \omega_{a}$, $\left.\omega_{0}+\mu \cdot \omega_{a}+\omega_{0}^{-1}\right)$. Für $\nu=0,1,2$ sei $T^{v}$ die topologische Summe aller $T_{\mathfrak{r}}$, $i \in I_{\text {, }}$. Ist $S_{0}$ eine diskrete Ordnung auf $I_{0}$, so ist $Q_{0}=\bigcup\left\{R_{i} \mid i \in I_{0}\right\} \cup$ $\cup \bigcup\left\{T_{i} \times T_{j} \mid i S_{0} j\right\}$ eine zulässige Ordnung auf $T^{0}$. Analog zeigt man, daß es für $\nu=1,2$ zulässige Ordnungen $Q_{\nu}$ auf $T^{\nu}$ gibt, wobei man nur bei $\nu=1$ aufpassen muß. Insbesondere kann man die $Q_{\dot{v}}$ stets so wählen, $\mathrm{da} \beta$ folgendes gilt:

1. Ist (c) erfüllt, so besitze $T^{1}$ bezüglich $Q_{1}$ einen End- aber keinen Anfangspunkt. Ist außerdem $I_{2} \neq \emptyset$, so besitze $T^{2}$ einen Anfangspunkt bezüglich $Q_{2}$.

2. Ist (d) erfüllt, (c) nicht erfüllt, so besitze $T^{2}$ keinen Anfangspunkt bezüglich $Q_{2}$.

Dann ist

$$
R=\bigcup_{=0}^{2} Q_{\nu} \cup\left(T^{0} \times T^{1}\right) \cup\left(T^{0} \times T^{2}\right) \cup\left(T^{1} \times T^{2}\right)
$$

eine zulässige Ordnung auf $T$.

Bezeichnet man die Vereinigungșmenge aller derjenigen Komponenten eines topologischen Raumes $T$, die wenigstens einen Randpunkt besitzen, mit $\boldsymbol{V}(T)$, so gilt:

SATZ 2a. Ein lokal-zusammenhängender Raum $T$ ist genau dann ordnungsfähig, wenn er randendlicher $T_{1}$-Raum ist und eine der folgenden Bedigungen erfüllt:

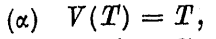

(ß) $\nabla(T)=\emptyset$,

( $\gamma) \quad V(T)$ ist nicht kompakt.

Beweis. a) Ist der lokal-zusammenhängende Raum $T$ durch $R$ zulässig geordnet, so ist er randendlicher $T_{1}$-Raum, Angenommen, es sei keine der Bedingungen $(\alpha)-(\gamma)$ erfüllt. Dann gibt es ohne Beschränkung der Allgemeinheit Elemente $x \in V(T), y \in T-V(T)$ mit $x R y$. Da $V(T)$ kompakt ist, hat die Menge $M=\{z \mid z \in V(T), z R y\}$ ein Maximum a.

Besäße $a$ keinen direkten Nachfolger in $T$, so wäre $a$ Häufungspunkt von $T-V(T)$ im Widerspruch zum lokalen Zusammenhang von $T$. Besäße $a$ einen direkten Nachfolger $b$, so wäre $b$ Randpunkt der $b$ enthaltenden Komponente von $T$, also Element von $V(T)$ im Widerspruch zur Maximaleigenschaft von $a$. Also ist obige Annahme falsch.

b) $T$ sei ein lokal-zusammenhängender, randendlicher $T_{1}$-Raum. Dann besitzt jede seiner Komponenten $K_{i}$ nach Satz 1 eine zulässige Ordnung $R_{i}$ und $T$ ist die topologische Summe der $K_{i}$. Erfüllt $T$ die Bedingung $(\alpha)$, so erfüllt er die Bedingung (a) von H.S. 9 ist also ordnungsfähig. Analog folgt aus ( $\beta$ ), daß die Bedingung (b) und aus $(\gamma)$, daß eine der Bedingungen (c), (d) von H.S. 9 erfüllt ist.

\section{Literaturverzeichnis}

[1] N. Bourbaki, Topologie générale, Ch. III, IV, 3e éd., Paris 1960.

[2] S. Eilenberg, Ordered topological spaces, Am. J. Math. 63 (1941). S. 39-45.

[3] B. Knaster und C. Kuratowski, Sur les ensembles connexes, Fund. Math. 2 (1921), S. 206-255.

[4] H. J. K o walsky, Kennzeichnung von Bogen, Fund. Math. 46 (1958), S. 103-107.

Regu par la Rédaction le 2\%. 11. 1964 